\title{
Interval Forecasting of Carbon Futures Prices Using a Novel Hybrid Approach with Exogenous Variables
}

\author{
Lu Zhang, ${ }^{1}$ Junbiao Zhang, ${ }^{1}$ Tao Xiong, ${ }^{1}$ and Chiao $\mathrm{Su}^{2}$ \\ ${ }^{1}$ College of Economics and Management, Huazhong Agricultural University, Wuhan 430070, China \\ ${ }^{2}$ Department of Agricultural and Resource Economics, University of California, Davis, CA, USA \\ Correspondence should be addressed to Tao Xiong; taoxiong@mail.hzau.edu.cn
}

Received 7 April 2017; Accepted 5 July 2017; Published 9 August 2017

Academic Editor: Luisa Di Paola

Copyright (c) 2017 Lu Zhang et al. This is an open access article distributed under the Creative Commons Attribution License, which permits unrestricted use, distribution, and reproduction in any medium, provided the original work is properly cited.

\begin{abstract}
This paper examines the interval forecasting of carbon futures prices in one of the most important carbon futures market. Specifically, the purpose of this study is to present a novel hybrid approach, which is composed of multioutput support vector regression (MSVR) and particle swarm optimization (PSO), in the task of forecasting the highest and lowest prices of carbon futures on the next trading day. Furthermore, we set out to investigate if considering some potential predictors, which have strong influence on carbon futures prices, in modeling process is useful for achieving better prediction performance. Aiming at testing its effectiveness, we benchmark the forecasting performance of our approach against four competitors. The daily interval prices of carbon futures contracts traded in the Intercontinental Futures Exchange from August 12, 2010, to November 13, 2014, are used as the experiment dataset. The statistical significance of the interval forecasts is examined. The proposed hybrid approach is found to demonstrate the higher forecasting performance relative to all other competitors. Our application offers practitioners a promising set of results with interval forecasting in carbon futures market.
\end{abstract}

\section{Introduction}

Forecasting carbon futures prices is part of the basis of financial investment decisions. Accurately forecasting carbon futures prices proves to be a great challenge and thus is of great interest to market stakeholders. Over the past decades, methods for forecasting carbon futures prices have attracted the attention of financial researchers and practitioners.

After an extensive review of the extant literature, we found that in recent years great research efforts have been expended in two areas: (1) understanding the underlying mechanisms that determine carbon futures prices $[1-3]$ and (2) the development of various models suitable for forecasting carbon futures prices [4-15]. A slight significant progress in forecasting carbon futures prices is notable. Specifically, Fan et al. [6] proposed a short-term prediction model, based on neural networks, for carbon futures prices forecasting. To capture the effects of various institutional features of carbon price, Koop and Tole [8] used dynamic model averaging to model and forecast spot and future prices in the EUETS carbon market. Zhu and Wei [11] developed a novel hybrid prediction model that exploits the unique strength of the ARIMA and LSSVM techniques for carbon futures prices forecasting. Hong et al. [13] developed a predictive regression model of carbon price movements with past returns of various commodities and financial products. Atsalakis [15] proposed three computational intelligence techniques, that is, a hybrid neurofuzzy controller that forms a closed-loop feedback mechanism, an artificial neural network based system, and an adaptive neurofuzzy inference system for accurately forecasting the changes in the carbon price. Nevertheless, it should be noted that the studies aforementioned concentrated on point forecasting instead of an interval one.

Interval forecasting of carbon futures prices has several advantages compared to point forecasting. Interval forecasting emphasizes the uncertainty and thus reduces the random variation, compared to the common single-value time series (e.g., carbon futures daily settlement prices). Interval forecasting of time series was established on the frontier of the literature following the work of Moore [16]. After establishing 
the basis for interval analysis in [16], Moore [17] further developed a set of mathematically rigorous error analysis techniques, for computational results. Other notable works in interval-valued time series (ITS) forecasting research include Arroyo et al. [18], who developed three exponential smooth methods for interval-valued time series, and Billard and Diday [19], who provided a method of fitting a linear regression model to interval-valued time series. These studies have spawned a growing body of literature with various applications in the fields of interval forecasting of time series. Successful applications include the finance market [20-23] and the energy market [24-27]. For example, Arroyo et al. [20] reviewed and compared the forecasting performance of exponential smoothing, ARIMA, multilayer perceptron, $k$ $\mathrm{NN}$, and vector error correction model in financial intervalvalued time series forecasting. Following the hybrid linear and nonlinear, Xiong et al. [25] proposed a novel modeling framework integrating bivariate empirical mode decomposition and support vector regression, extended from the wellestablished empirical mode decomposition based time series modeling framework, for interval forecasting of electricity demand. While these studies have many contributions, we have identified some of the limitations in the current technique. For example, potential variables that may affect interval-valued time series are ignored in these studies, which tend to depreciate the modeling quality as well as overall prediction performance.

This study focuses on interval forecasting of carbon futures prices with consideration of a large set of potential predictors. Generally speaking, two fundamental issues must be addressed to achieve this objective: feature selection and modeling technique. As Koop and Tole [8] pointed out, a large number of potential variables may affect carbon futures prices. Thus, several potential predictors, which have also been considered in other studies, are evaluated in this study. A filter method, in which the predictor subset is chosen by an evaluation criterion defined in advance, based on the dataset is employed for feature selection in this study. This evaluation criterion should be able to measure the relationship of each subset of predictors with the two outputs (i.e., the highest and lowest prices of carbon futures). Therefore, 2 -fold crossvalidation of a 1-NN approximator [28] is adopted for feature selection here.

The presence of many predictors is bound to place a high demand for the generalization ability of modeling technique. As a novel intelligent algorithm, multioutput support vector regression (MSVR), which is the generalization of the standard SVR, has attracted particular attention from a variety of disciplines. It has especially been successful when applied to interval forecasting that is of multioutput modeling problem in essence. To address the interval forecasting of carbon futures prices with a variety of predictors, the MSVR is selected as the modeling technique here. In addition, the generalization ability of the MSVR depends on adequately setting parameters. Therefore, particle swarm optimization (PSO) developed by Kennedy and Eberhart [29] is used for parameter selection of MSVR for interval forecasting. Thus, we propose a novel hybrid prediction method (abbreviated as MSVR-PSO) by incorporating MSVR and PSO for interval forecasting of carbon futures prices. More importantly, we further investigate the feasibility of forecasting the two bounds of carbon futures prices series simultaneously by MSVR-PSO with influencing variables (abbreviated as MSVR-PSO-W). In this MSVR-PSO-W model, the inputs are the potential predictors, while the outputs correspond to the forecasts of the two bounds.

For comparison purposes, the MSVR-PSO model without influencing variables is constructed and selected as benchmark (abbreviated as MSVR-PSO-WO). In addition, two traditional ITS modeling techniques, Holt's exponential smoothing method for intervals (Holt ${ }^{\mathrm{I}}$ ) [30] and vector error correction model (VECM) [31], are also selected as benchmarks. It should be noted that, in the cases of Holt ${ }^{\mathrm{I}}$ and VECM, only historical lower and upper bounds of carbon futures prices (without influencing variables) are used as predictors. Another traditional intelligent algorithm, artificial neural networks (ANN), is also selected as benchmarks. More specifically, two artificial neural networks-based prediction models, with and without influencing variables, are constructed (abbreviated as ANN-W and ANN-WO, resp.). The largest carbon market, European Union emissions trading scheme (EUETS), is chosen as the experimental datasets. Evaluation of the proposed model and selected counterparts is performed using out-of-sample forecasts.

In summary, for the purpose of constructing models that can accurately perform interval forecast of daily carbon futures prices, the contributions of this study are presented as follows. First, we proposed a MSVR-based prediction model for interval forecasting of carbon futures prices. Experimental results indicate that the proposed prediction model is a promising alternative for interval forecasting of carbon futures prices. Second, we investigate whether considering a large set of potential influencing variables is useful to achieve a better prediction performance for interval forecasting. Third, the feasibility of forecasting the two bounds of intervalvalued carbon futures prices series simultaneously by the MSVR with influencing variables is examined. Finally, we provide the empirical evidence on the interval forecasting of carbon futures prices forecasting with real-world data from European carbon futures market.

The rest of the article is organized as follows. In Section 2, the construction of an interval-valued carbon futures prices series and the description of the proposed MSVR-PSO method are detailed. Section 3 discusses the research design and experimental results. Section 4 provides the conclusion.

\section{Forecasting Method for Interval-Valued Carbon Futures Price}

2.1. Construction of Interval-Valued Carbon Futures Prices. An interval-valued variable, $\mathbf{X}_{t}=\left\{\left[X_{t}^{L}, X_{t}^{U}\right]^{T}: X_{t}^{L}, X_{t}^{U} \in\right.$ $\left.\mathfrak{R}, X_{t}^{L} \leq X_{t}^{U}\right\}, \forall t \in E$, is a variable where each element is an interval instead of a single point. Let the $t$ th element of $X$ be $X_{t}$. Let $X_{t}$ be represented as an interval, with its lower and upper bounds, $\mathbf{X}_{t}=\left[X_{t}^{L}, X_{t}^{U}\right]^{T}$. The daily values of the carbon futures prices at EUETS can be represented as interval values, as shown in Table 1. 
TABLE 1: Interval-valued variable.

\begin{tabular}{lc}
\hline Year 2014 & $\begin{array}{c}\text { Carbon futures prices (Euro cents per tonne) } \\
\text { [Lower, upper] }\end{array}$ \\
\hline Oct. 09 & {$[5.98,6.15]$} \\
Oct. 10 & {$[5.96,6.11]$} \\
Oct. 13 & {$[5.86,6.07]$} \\
Oct. 14 & {$[5.99,6.18]$} \\
Oct. 15 & {$[6.01,6.17]$} \\
Oct. 16 & {$[6.13,6.24]$} \\
Oct. 17 & {$[6.12,6.27]$} \\
$\ldots$ & $\ldots$ \\
$\ldots$ & $\ldots$ \\
\hline
\end{tabular}

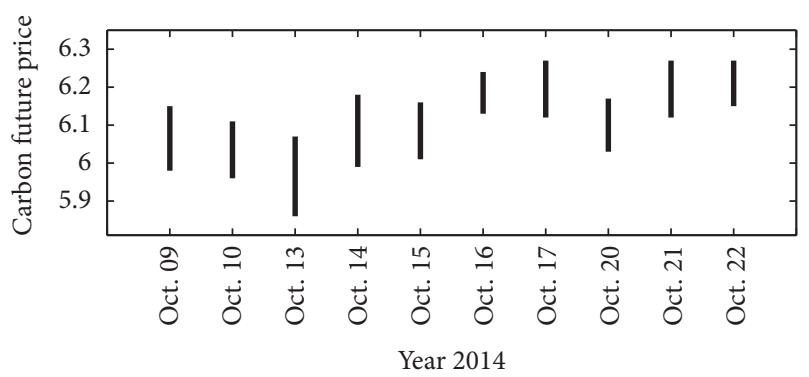

FIGURE 1: Daily interval-valued carbon futures prices.

The daily carbon futures prices form a sequence of interval-valued variables, $\mathbf{X}_{t}=\left[X_{t}^{L}, X_{t}^{U}\right]^{T}$ for $t=1, \ldots, n$, with lower bound $X_{t}^{L}$ and upper bound $X_{t}^{U}$. Figure 1 depicts the set of daily carbon futures prices from October 09, 2014, to October 22, 2014.

\subsection{MSVR for Interval-Valued Carbon Futures Prices Forecast-} ing. Multioutput support vector regression (MSVR) technique for interval forecasting of carbon futures prices is briefly described in this subsection. For more in-depth discussion on MSVR, please refer to [32-34]. MSVR, a generalization of the standard SVR, is originally developed by Tuia et al. [34] to solve the nonlinear regression problem for multiple variables. Interval forecasting is a multioutput modeling problem, and MSVR has been successfully used for interval forecasting $[25,35]$.

Assume there is an interval-valued carbon futures price $\left[X_{t}\right]=\left[X_{t}^{L}, X_{t}^{U}\right]^{T}$ for $t=1, \ldots, n$ as shown in Figure 1 , where $X_{t} \in \mathfrak{R}^{2}$ is the $t$ th interval. The basic idea of interval forecasting using MSVR is to find the mapping between an input vector and an output vector from a given dataset $\left\{\left(\mathbf{x}_{i}, \mathbf{y}_{i}\right)\right\}_{i=d}^{n}$, where $d$ denotes embedded dimension. The input vector, $\mathbf{x}=\left[X_{i}^{L}, X_{i}^{U}, X_{i-1}^{L}, X_{i-1}^{U}, \ldots, X_{i-d+1}^{L}, X_{i-d+1}^{U}, P_{i}^{1}\right.$, $\left.P_{i}^{2}, \ldots, P_{i}^{m}\right]^{T} \in \mathfrak{R}^{2 d+m}$, is composed of the lagged intervals of carbon futures prices from period $i$, and the set of influencing variables at the same period. The output vector, $\mathbf{y}=\left[X_{i+1}^{L}, X_{i+1}^{U}\right]^{T} \in \mathfrak{R}^{2}$, is the interval of next period carbon futures price. Thus, our MSVR model contains $2 d+m$ inputs and two outputs, corresponding to the forecast of the two bounds, $X_{i+1}^{L}$ and $X_{i+1}^{U}$, of interval-valued carbon futures prices.

The MSVR solves the stated mapping problem by finding the regressors $\mathbf{w}^{j}$ and $b^{j}(j=1,2)$ for every output that minimizes the following equation:

$$
L_{p}(\mathbf{W}, \mathbf{b})=\frac{1}{2} \sum_{j=1}^{2}\left\|\mathbf{w}^{j}\right\|^{2}+C \sum_{i=1}^{n} L\left(u_{i}\right),
$$

where

$$
\begin{aligned}
u_{i} & =\left\|\mathbf{e}_{i}\right\|=\sqrt{\left(\mathbf{e}_{i}^{T} \mathbf{e}_{i}\right)}, \quad \mathbf{e}_{i}^{T}=\mathbf{y}_{i}^{T}-\varphi\left(\mathbf{x}_{i}\right) \mathbf{W}-\mathbf{b}^{T}, \\
\mathbf{W} & =\left[\mathbf{w}^{1}, \mathbf{w}^{2}\right], \\
\mathbf{b} & =\left[b^{1}, b^{2}\right]^{T},
\end{aligned}
$$

where $\varphi(\cdot)$ is a nonlinear transformation of the feature space. $C$ is the parameter that represents the trade-off between the regularization and error reduction terms, which will be tuned by particle swarm optimization. $L(u)$ is a quadratic epsilon-insensitive cost function defined in (3), which is a differentiable form of the Vapnik $\varepsilon$ insensitive loss function

$$
L(u)= \begin{cases}0 & u<\varepsilon \\ u^{2}-2 u \varepsilon+\varepsilon^{2} & u \geq \varepsilon .\end{cases}
$$

When $\varepsilon$ is nonzero in (3), the function will take all outputs into account when constructing each individual regressor and will obtain more robust predications and will then yield a single support vector set for all dimensions. Noteworthy, the proposed optimization problem can be resolved by an iterative reweighted least squares (IRWLS) procedure developed by Sanchez-Fernandez et al. [33]. The objective of (1) can be approximated as (4) by means of firstorder Taylor expansion of the cost function $L(u)$

$$
\begin{aligned}
L_{p}^{\prime}(\mathbf{W}, \mathbf{b})=\frac{1}{2} \sum_{j=1}^{2}\left\|\mathbf{w}^{j}\right\|^{2}+\frac{1}{2} \sum_{i=1}^{n} a_{i} u_{i}^{2}+\mathrm{CT}, \\
a_{i}= \begin{cases}0 & u_{i}^{k}<\varepsilon \\
\frac{2 C\left(u_{i}^{k}-\varepsilon\right)}{u_{i}^{k}} & u_{i}^{k} \geq \varepsilon,\end{cases}
\end{aligned}
$$

where CT is a constant term that does not depend on $\mathbf{W}$ and b and the superscript $k$ denotes the $k$ th iteration.

An IRWLS procedure, which linearly searches the next step solution along the descending direction based on the previous solution [33], is constructed to solve the minimization problem of (4). Based on the Representer Theorem [36], $\mathbf{w}^{j}=\sum_{i} \phi\left(\mathbf{x}_{i}\right) \boldsymbol{\beta}^{j}=\boldsymbol{\Phi}^{T} \boldsymbol{\beta}^{j}$ is the best solution of (4) in the feature space. Thus, the objective of the MSVR problem is transformed to search the objective minimizing $\beta$ and $\mathbf{b}$.

The general steps of the IRWLS procedure can be described as follows. For detailed description of IRWLS, 
TABLE 2: The statistical description of the interval-valued carbon future prices.

\begin{tabular}{lccccccc}
\hline Bound & Sample size & Min & Max & Mean & Std & Skewness & Kurtosis \\
\hline Upper bound & 1101 & 3.20 & 21.25 & 9.6868 & 5.15093 & 0.801 & -0.806 \\
Lower bound & 1101 & 15050 & 33300 & 20903.96 & 5.21888 & 0.820 & -0.774 \\
\hline
\end{tabular}

Notes. The minimum, maximum, and standard deviation are given under the Mix, Max, and Std, respectively.

please refer to [32,33]. The first step initializes the parameters as $k=0, \boldsymbol{\beta}^{k}=0$, and $\mathbf{b}^{k}=0$ and then computes $u_{i}^{k}$ and $a_{i}$. The second step is to calculate the solutions $\boldsymbol{\beta}^{s}$ and $\mathbf{b}^{s}$ according to $\left[\begin{array}{cc}\mathbf{K}+\mathbf{D}^{-1} & 1 \\ \mathbf{a}^{T} \mathbf{K}^{a} & 1^{T} \mathbf{a}\end{array}\right]\left[\begin{array}{c}\boldsymbol{\beta}^{j} \\ b^{j}\end{array}\right]=\left[\begin{array}{c}\mathbf{y}^{j} \\ \mathbf{a}^{T} \mathbf{y}^{j}\end{array}\right], j=1,2$, where $\mathbf{a}=\left[a_{1}, \ldots, a_{n}\right]^{T},\left(\mathbf{D}_{a}\right)_{i j}=a_{i} \delta(i-j)$, and $\mathbf{K}$ is the kernel matrix. The corresponding descending direction is defined as $\mathbf{p}^{k}=\left[\begin{array}{c}\mathbf{w}^{s}-\mathbf{w}^{k} \\ \left(\mathbf{b}^{s}-\mathbf{b}^{k}\right)^{T}\end{array}\right]$. The third step is to calculate $\boldsymbol{\beta}^{k+1}$ and $\mathbf{b}^{k+1}$ by using a backtracking algorithm and then obtain $u_{i}^{k+1}$ and $a_{i}$. Return to step 2 until the function converges.

In this study, the radial basis function (RBF) is selected as the kernel function for MSVR. As such, three hyperparameters, namely $C, \varepsilon$, and $\sigma$, of MSVR will need to be determined. Here, a PSO-based method for this parameter selection of MSVR is developed and detailed in the following subsection.

2.3. PSO for Parameter Selection of MSVR. Particle swarm optimization (PSO) developed by Kennedy and Eberhart [29] is an emerging population-based evolutionary algorithm inspired by social behavior among individuals. PSO performs searches on the basis of a population (swarm) of several individuals (particles). Each particle represents a candidate position (potential solution) and moves in an $\mathrm{N}$-dimension search space.

Given an $N$-dimension search space, let $p_{i}^{t}=$ $\left\{p_{i 1}^{t}, p_{i 2}^{t}, \ldots, p_{i N}^{t}\right\}$ and $v_{i}^{t}=\left\{v_{i 1}^{t}, v_{i 2}^{t}, \ldots, v_{i N}^{t}\right\}$ denote the position and velocity of the particle $i$ at iteration $t$, respectively. To find the optimal solution, each particle updates its position according to two experiences, its own best previous solution (pbest) and the best solution in swarm (gbest) until iteration $t$. Each particle updates its velocity according to the following equation:

$$
\begin{array}{r}
v_{\text {in }}^{t}=v_{\text {in }}^{t-1}+c_{1} r_{1}\left(\text { pbest }_{i}^{t}-p_{\text {in }}^{t}\right)+c_{2} r_{2}\left(\text { gbest }^{t}-p_{\text {in }}^{t}\right), \\
n=1,2, \ldots, N,
\end{array}
$$

where $c_{1}$ and $c_{2}$ denote the cognition and social learning factors, respectively, and $r_{1}$ and $r_{2}$ are both random variables, uniformly distributed in $U(0,1)$. After obtaining the new velocity, each particle updates its position as follows:

$$
x_{i n}^{t+1}=x_{i n}^{t}+v_{i n}^{t}, \quad n=1,2, \ldots, N .
$$

In this study, we develop a MSVR-PSO method, in which PSO is used to solve the parameter for MSVR, for interval forecasting of carbon futures prices. As discussed in Section 2.2, three hyperparameters, the decision variables $C, \varepsilon$, and $\sigma$, are required and are tuned in a 3-dimensional search

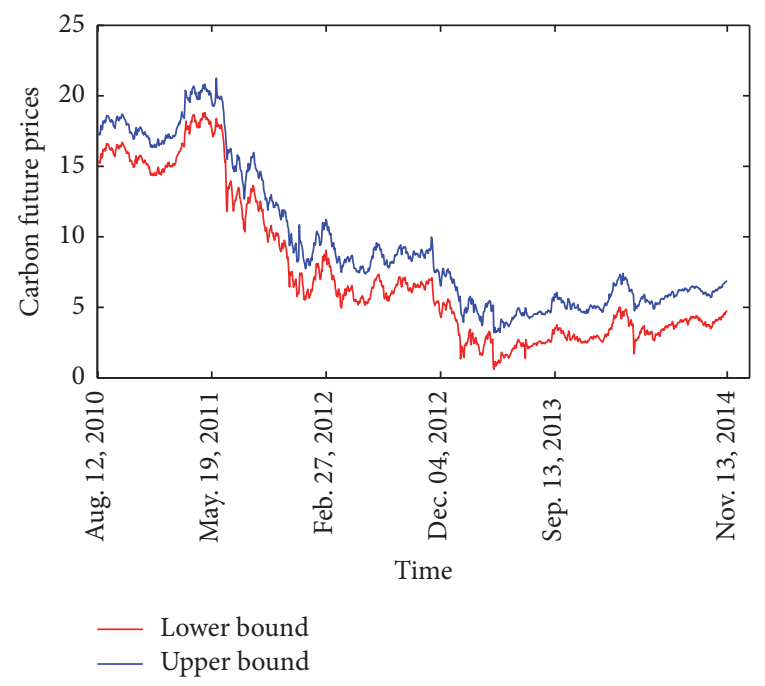

FIGURE 2: Interval-valued carbon futures prices series.

space. The search space of these parameters is exponentially growing: $\log _{2} C \in[-6,6], \log _{2} \varepsilon \in[-6,6]$, and $\log _{2} \sigma \in$ $[-6,6]$.

The fitness function of each particle is defined as the prediction performance of the MSVR model in the interval forecasting of carbon futures price, in terms of the interval $U$ of Theil statistic $\left(U^{\mathrm{I}}\right)$. This is a classic accuracy measure and has been widely adopted for interval forecasting [22, 30, 35]. The definition of $U^{\mathrm{I}}$ is as follows:

$$
U^{\mathrm{I}}=\sqrt{\frac{\sum_{t=1}^{n}\left(X_{t+1}^{U}-\widehat{X}_{t+1}^{U}\right)^{2}+\sum_{t=1}^{n}\left(X_{t+1}^{L}-\widehat{X}_{t+1}^{L}\right)^{2}}{\sum_{t=1}^{n}\left(X_{t+1}^{U}-X_{t}^{U}\right)^{2}+\sum_{t=1}^{n}\left(X_{t+1}^{L}-X_{t}^{L}\right)^{2}}}
$$

where $n$ is the amount of fitted intervals and $\left[X_{t}^{L}, X_{t}^{U}\right]$ and $\left[\widehat{X}_{t}^{L}, \widehat{X}_{t}^{U}\right]$ are the $t$ th true and fitted intervals, respectively.

\section{Empirical Analysis}

3.1. Data Descriptions and Input Selection. The ECX EUA future prices used in our empirical analysis are obtained from the Intercontinental Futures Exchange. The sample data are daily carbon futures prices traded in the European Union, covering the period from August 12, 2010, to November 13, 2014, with a total of 1101 observations, as shown in Figure 2. To improve visibility, the lower bound of carbon future prices in Figure 2 is the actual lower bound minus 2. The summary statistics of sample data are presented in Table 2. 


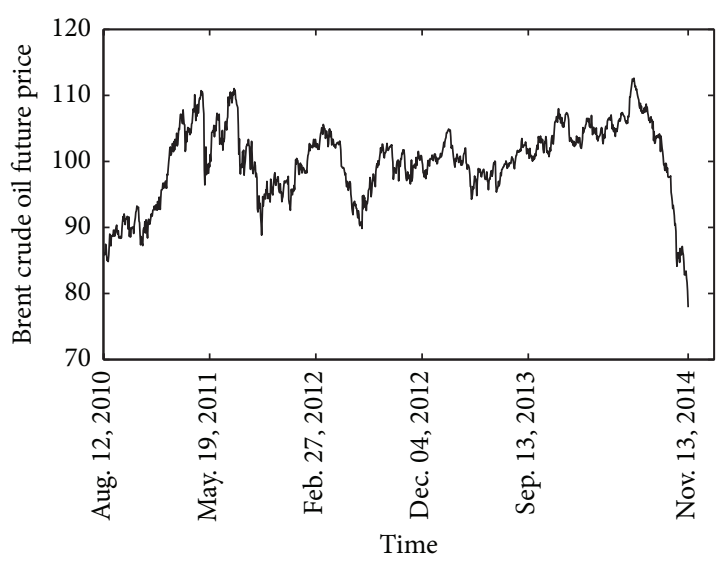

Figure 3: Brent crude oil future prices series.

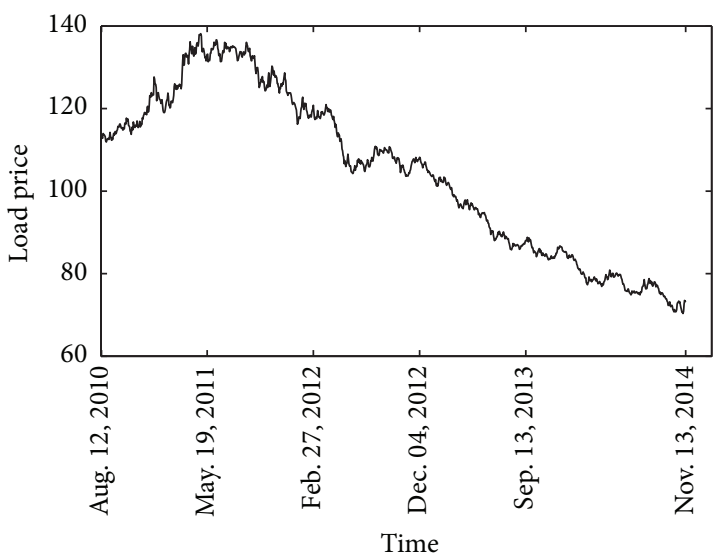

FIGURE 4: Load price series.

As discussed in Section 1, this study attempts to investigate whether considering a large set of potential influencing variables can achieve a better prediction performance for interval forecasting. The set of potential influencing variables chosen for this study is specified as below.

(i) Oil Price. The Brent crude oil futures prices (euros per barrel) are selected because this is the most important marker for European oil and is a decisive factor in the configuration of prices of carbon and other commodities $[8,37,38]$. The daily settlement prices of Brent crude oil future are taken from the Intercontinental Commodities Exchange, as shown in Figure 3.

(ii) Load Price. Following the studies of [8, 37, 39], the daily load prices, measured as the Phelix base and collected from the European Energy Exchange, are selected as a predictor. The load price series considered in this study is shown in Figure 4.

(iii) Gas Price. Natural gas futures prices are selected as a predictor [8]. We referred to gas futures traded in United Kingdom, which is one of Europe's largest exchange. The daily settlement prices of natural gas futures are available

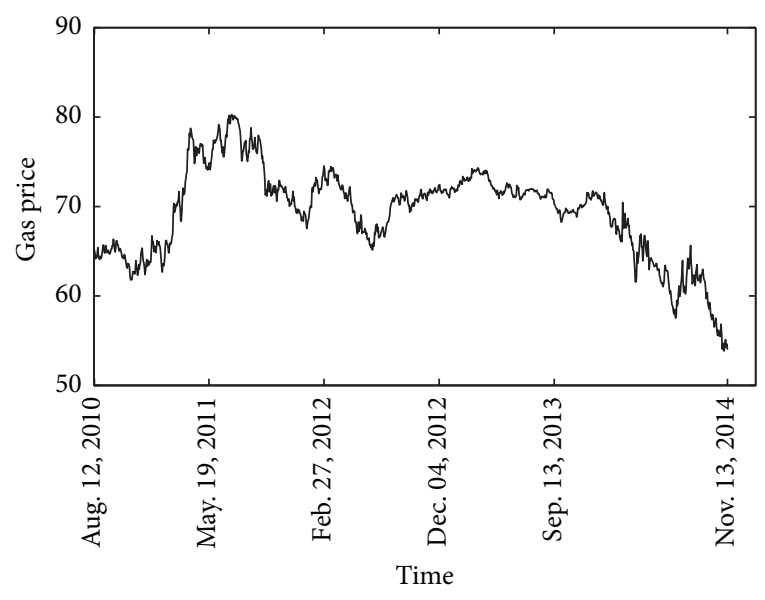

FIGURE 5: Natural gas futures prices series.

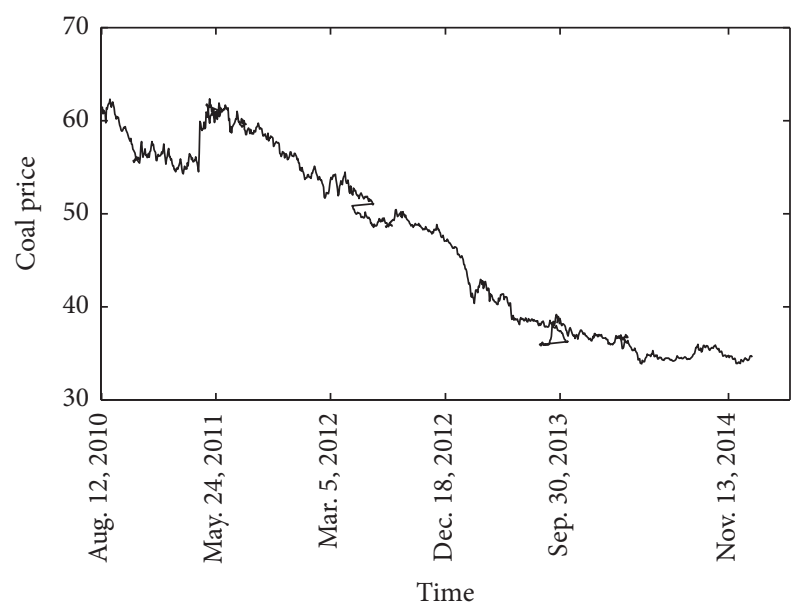

Figure 6: Coal price series.

from the Intercontinental Commodities Exchange, as shown in Figure 5.

(iv) Coal Price. Coal prices, specifically the coals delivered to Amsterdam, Rotterdam, and Antwerp region in the Netherlands, are selected in this study. Coal prices are included because they may affect carbon prices by means of the fuel switching behavior of electricity-generating plants $[8,38]$. The daily settlement prices of coal are available from the Stevens Reference Futures, as shown in Figure 6.

(v) Temperature. Following studies from [8, 37, 38], we include the temperature in Europe as a predictor. The daily temperature of 14 Europe countries was obtained from European climate assessment, as shown in Figure 7. Then the weighted average across countries is calculated, in which the weights were proportional to the annual population. Finally, the absolute value of the deviation from this weighted average temperature was computed.

(vi) Stock Price. The daily settlement prices of Euronext 100 index are chosen in this study, as shown in Figure 8. 


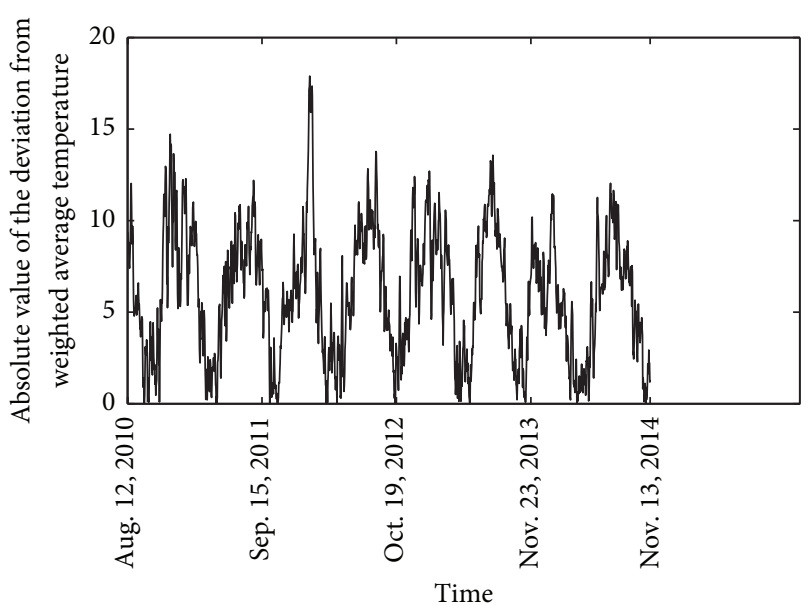

Figure 7: Temperature series.

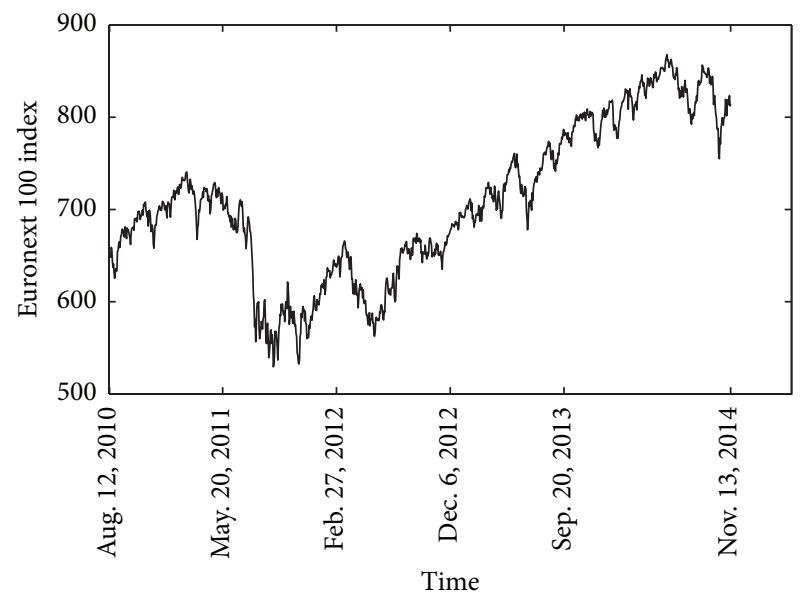

Figure 8: Euronext 100 index series.

They are the blue chip index of the Pan-European Exchange and are comprised of the largest and most liquid stocks traded on the Euronext. Given that the stock market is an important indicator of the health of the economy, and economic growth is expected to raise the demand for energy, and thus for carbon permits [8], stock performance is selected as a predictor for carbon futures prices.

The historical intervals of carbon futures prices, and the potential variables stated above, are chosen as variables that may affect carbon futures prices. These variables are the inputs for the model in this study. The maximum embedding order for historical intervals, $d$, is set to 10. The initial input variables are $\left[X_{i}^{L}, X_{i}^{U}, X_{i-1}^{L}, X_{i-1}^{U}, \ldots\right.$, $\left.X_{i-d+1}^{L}, X_{i-d+1}^{U}, P_{i}^{1}, P_{i}^{2}, \ldots, P_{i}^{m}\right] \in \mathfrak{R}^{2 d+m}, d=10$, and $m=6$. The best subset of inputs is then identified using the filter method. As discussed in Section 1, the 2-fold cross-validation of a 1-NN approximator [28] is selected as evaluation criteria in the filter method. The search algorithm used in the filter method is a forward-backward selection method.
TABLE 3: Parameter selection of the PSO.

\begin{tabular}{lc}
\hline Parameters & Values \\
\hline Swarm size & 30 \\
Number of iterations & 400 \\
Cognitive coefficients & 2.0 \\
Interaction coefficients & 2.0 \\
Initial weight & 0.9 \\
Final weight & 0.4 \\
\hline
\end{tabular}

3.2. Statistical Criteria and Methodologies Implementation. To evaluate and compare the effectiveness of the proposed prediction models with selected counterparts, two commonly used statistical criteria are adopted here. First, the forecast accuracy of all estimated models is examined in terms of $U^{\mathrm{I}}$, which is defined in (7). Second, we use the analysis of variance (ANOVA) test to investigate if the means of the accuracy measure (i.e., $U^{\mathrm{I}}$ ) are significantly different among the six prediction models. If so, Tukey's honesty significant difference (HSD) [40] tests are then applied to further identify the significantly different models by means of multiple pairwise comparisons.

As discussed in Section 1, two interval-valued forecasting techniques, that is, Holt ${ }^{\mathrm{I}}$ and VECM, and two ANN-based prediction models with and without influencing variables (ANN-W and ANN-WO) are constructed and selected as benchmarks. To justify whether considering the influencing variables is useful for achieving better prediction performance, a MSVR-PSO model without the influencing variables (MSVR-PSO-WO) is also chosen as a benchmark. Detailed formulations of these selected methods (Holt ${ }^{\mathrm{I}}$, VECM, and ANN) can be found in [30,31].

The proposed MVSR-PSO prediction model is implemented in MATLAB. Specifically, the MSVR with two outputs is implemented using the program package developed by Pérezcruz et al. [32]. The PSO is implemented in MATLAB with the formulation presented by Kennedy and Eberhart [29]. Certain parameters in the PSO, for example, swarm size, number of iterations, cognitive coefficient, and interaction coefficient, should be determined in advance. By doing so, all the particles can be randomly generated among the search space discussed in Section 2.3. All the velocity components are assigned the initial value of 0 . The parameters of PSO are determined in line with the recommendations in [41-44]. The final parameters of PSO are shown in Table 3.

With respect to the VECM estimation, a preliminary analysis on the interval-valued carbon futures prices shown in Figure 2 is performed first. The augmented Dickey-Fuller (ADF) test results at the level of 0.05 are reported in Table 4. It is clear that both upper and lower bounds series are nonstationary in level but stationary in 1-first differences. The Johansen test is applied to examine any cointegrated relations between the upper bound $X_{i}^{U}$ and lower bound $X_{i}^{L}$. According to the results of cointegration test at the level of 0.05 as shown in Table 5, the null hypothesis of no cointegration is rejected, but the test fails to reject the hypothesis that at most one cointegration vector exists. These 
TABLE 4: ADF test results.

\begin{tabular}{lccr}
\hline Bound & Test for unit root in & $T$ & $p$ \\
\hline \multirow{2}{*}{ Upper bound } & Level & -1.760420 & 0.0744 \\
& 1st difference & $-11.95007^{* *}$ & 0.0000 \\
Lower bound & Level & -1.698380 & 0.0847 \\
& 1st difference & $-23.48374^{* *}$ & 0.0000 \\
\hline
\end{tabular}

Notes. T-statistics and $p$ value are given in the columns " $T$ " and " $p$. " ** denotes significance at the $5 \%$ level.

TABLE 5: Cointegration test results.

\begin{tabular}{lccccc}
\hline & Eigenvalue & Trace statistics & $p$ value & Low bound & Upper bound \\
\hline At most 1 & 0.002118 & 2.317761 & 0.1279 & & 7 \\
None & $0.054679^{* *}$ & $63.77838^{* *}$ & 0.0000 & & 7 \\
$C$ & & & & 1 & -1.012751 \\
\hline
\end{tabular}

Notes. "None" corresponds to the null hypothesis of no cointegration and "at most 1" corresponds to the hypothesis of at most one cointegration vector. The row labeled " $\mathrm{C}$ " gives cointegrating vectors with the coefficients of the lower bound series normalized to one. $* *$ denotes significance at the $5 \%$ level.

results confirm that the dimension of the cointegration space is one. As such, the upper and lower bounds series of the daily carbon futures prices from August 12, 2010, to November 13, 2014 , are considered to be $\mathrm{CI}(1,1)$. In light of these results, a VECM is constructed to further investigate the short-run and long-run interactions between the upper bound $X_{i}^{U}$ and lower bound $X_{i}^{L}$. The VECM for interval-valued carbon futures prices is implemented in Eviews.

The Holt ${ }^{\mathrm{I}}$ is implemented with the methods used by Maia and de Carvalho [30]. We estimate the smoothing parameter matrices with elements constrained to the rang $(0$, 1), by minimizing the interval sum of the squared forecasting errors. The limited memory BFGS method, which has been implemented in program package "optimx" ( $\mathrm{R}$ package “optimx" is available at http://ftp.ctex.org/mirrors/CRAN/) in $\mathrm{R}$ software, is adopted here to solve the optimization problem.

3.3. Experimental Results. In this section, we present the experiments carried out to examine the out-of-sample prediction performance of the proposed MSVR-PSO method using real-world datasets in carbon futures markets against some selected competitors.

Figure 9 illustrates the experimental procedure with the interval-valued carbon future prices. The carbon futures prices series described in Section 3.1 is split into an estimation sample and a hold-out sample. The first two-thirds of the observations are selected as estimation sample, while the remainder is saved for the hold-out sample. Each prediction method is trained in the estimation sample, and the prediction performance is evaluated in the hold-out sample. Afterward, the input selection is performed via filter method. As discussed in Section 3.1, the initial input variables are $\left[X_{i}^{L}, X_{i}^{U}, X_{i-1}^{L}, X_{i-1}^{U}, \ldots, X_{i-d+1}^{L}, X_{i-d+1}^{U}, P_{i}^{1}, P_{i}^{2}, \ldots, P_{i}^{m}\right] \in$ $\mathfrak{R}^{2 d+m}, d=10$, and $m=6$, where $X_{i}^{L}, X_{i}^{U}, X_{i-1}^{L}, X_{i-1}^{U}, \ldots$, $X_{i-d+1}^{L}, X_{i-d+1}^{U}$ denote the historical intervals of carbon futures prices at $i, i-1, \ldots$, and $i-9$, respectively. $P_{i}^{1}, P_{i}^{2}$, $\ldots, P_{i}^{6}$ denote the six influencing variables at $i$. To select the best subset of original inputs, the 2 -fold cross-validation of a 1-NN approximator is adopted as evaluation criteria, and the forward-backward selection method is employed as search algorithm. By doing so, the best subsets of original inputs for MSVR-PSO-W are as follows: $X_{i}^{L}, X_{i}^{U}, X_{i-1}^{L}$, $X_{i-2}^{L}, X_{i-2}^{U}, X_{i-4}^{L}, X_{i-6}^{U}, X_{i-7}^{L}, P_{i}^{2}$ (load price), $P_{i}^{3}$ (gas price), and $P_{i}^{4}$ (coal price). Meanwhile, the model selection is determined by the means of the methodologies implementation presented in Section 3.2 and a fivefold cross-validation technique in estimation sample. Finally, the interval $U$ of Theil statistic $\left(U^{\mathrm{I}}\right)$ is computed for each obtained prediction model in hold-out sample. The previous modeling process is repeated 50 times, which produces $50 U^{\mathrm{I}}$ for each prediction model. Upon the termination of this loop, the prediction performance of different methods considered in this study is examined and compared in terms of the mean of $U^{\mathrm{I}}$ of the 50 replications in hold-out sample.

The prediction performance of the six examined methods, that is, MSVR-PSO-W, MSVR-PSO-WO, ANN-W, ANNWO, Holt ${ }^{\mathrm{I}}$, and VECM, in terms of $U^{\mathrm{I}}$ is shown in Figure 10. To improve visibility, in addition, Figure 11 depicts representative example of actual intervals versus predicted intervals from September 8, 2014, to October 22, 2014. One can deduce the following understandings according to the experimental results.

(i) Overall, the top four methods, MSVR-PSO-W, MSVR-PSO-WO, ANN-W, and ANN-WO, are almost in a tie. Obviously, the proposed MSVR-PSO$\mathrm{W}$ method achieves the best performance relative to all of the other competitors.

(ii) MSVR-PSO-W forecasts more accurately than MSVR-PSO-WO (though only marginally). Perhaps 


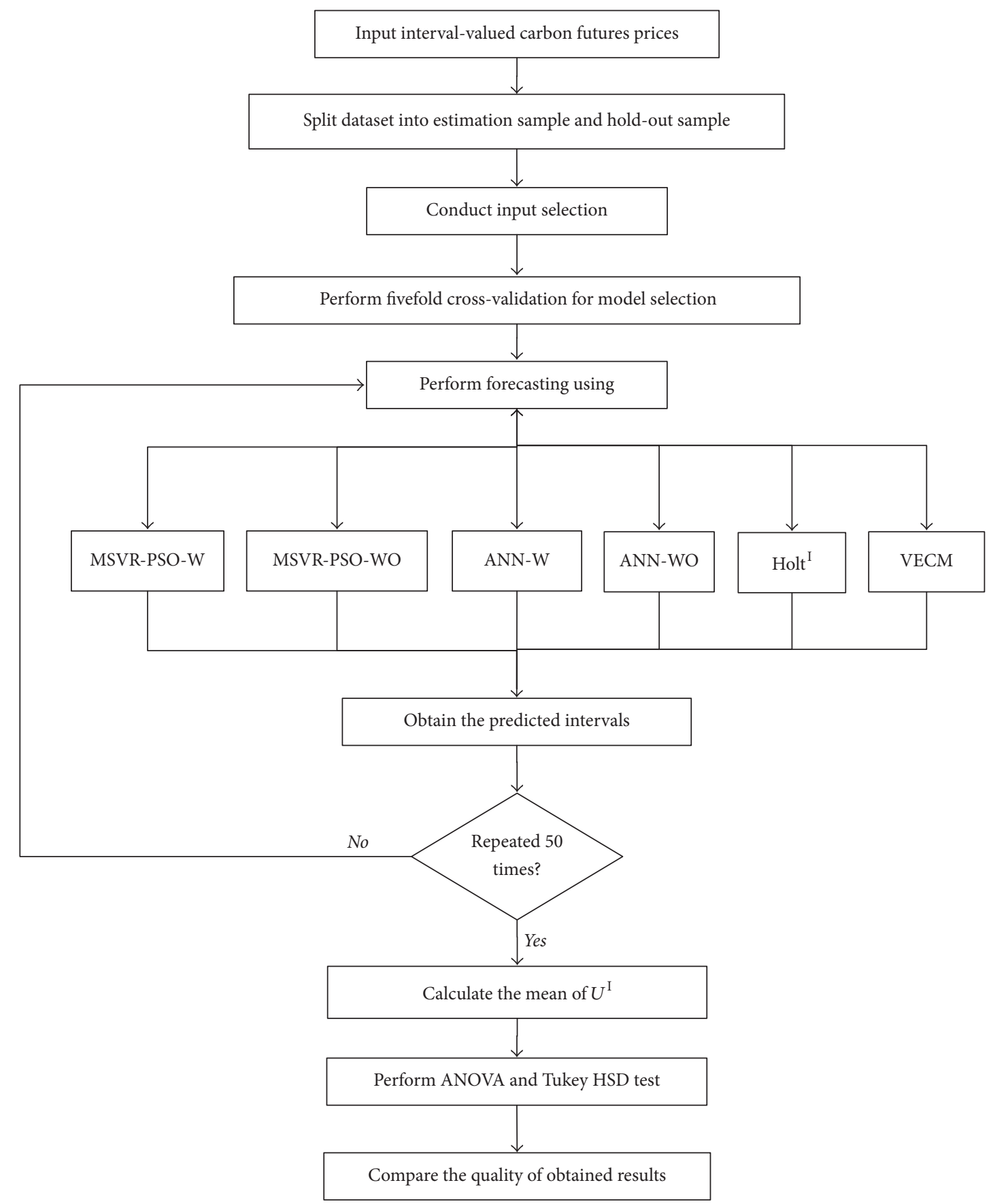

FIGURE 9: Experimental procedure.

the reason for the superiority of MSVR-PSO$\mathrm{W}$ method is because it brings some potential predictors, which have strong influence on the carbon futures prices, into the inputs in modeling process. Thus, further proof of the superiority of considering potential influencing factors in interval forecasting is provided in this study.

(iii) Comparing the two MSVR-based prediction models (MSVR-PSO-W and MSVR-PSO-WO) and the two
ANN-based prediction models (ANN-W and ANNWO), the former are generally better. Perhaps the reason for the superiority of MSVR relative to ANN is that MSVR, which uses structural risk minimization, is less prone to overfitting.

(iv) As far as the comparison between ANN-W and ANNWO is concerned, the ANN-W is slightly better. This ranking further attests to the value that is added by considering potential influencing factors in interval forecasting. 


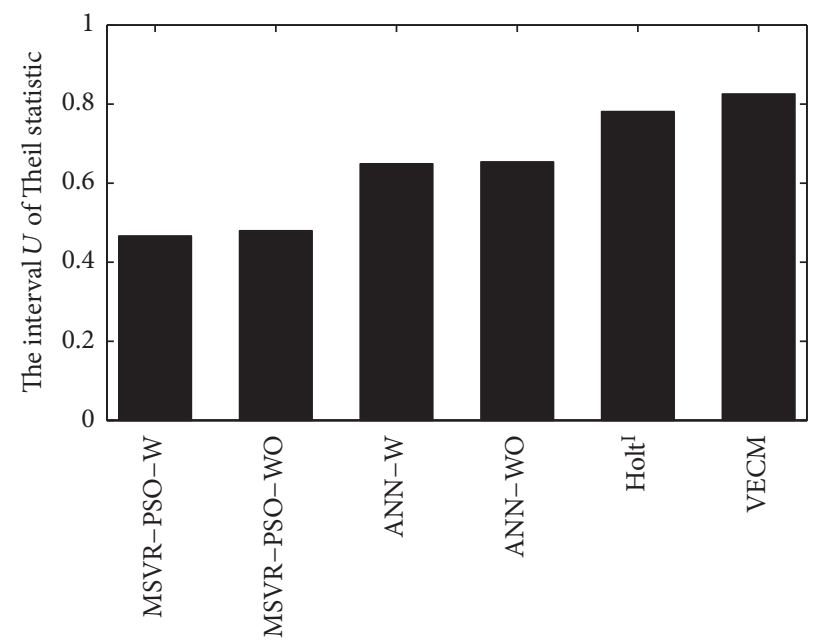

FIGURE 10: Prediction performance of examined methods in terms of $U^{\mathrm{I}}$.

(v) Two traditional ITS methods (Holt ${ }^{\mathrm{I}}$ and VECM) almost have the worst performance. It is conceivable that the reason of the inferiority of the $\operatorname{Holt}^{\mathrm{I}}$ and VECM for ITS forecasting is that these can provide good predictions only when ITS under study is linear and stationary. But the interval-valued carbon futures prices series examined in this study unfortunately appears to be obviously nonlinear and nonstationary as shown in Figure 2.

Next, an ANOVA procedure is performed to identify if the means of the accuracy measure (i.e., $U^{\mathrm{I}}$ ) are significantly different at a significance level of 0.05 among the six prediction models. The $F$ statistic and corresponding $p$ value of ANOVA test are 1067.11 and 0.000 , respectively, indicating that there are significant differences among the six prediction models. As such, Tukey's honesty significant difference (HSD) test is then employed to further investigate the significant difference between any two models here. The multiple comparison test results at the significance level of 0.05 are reported in Table 6 in the form of a five-column matrix. Each row of the matrix represents one test, and there is one row for each pair of groups. The entries in the row indicate the two models being compared, the difference in means of $U^{\mathrm{I}}$ of two models compared, and a confidence interval (CI) for the difference. For each row of Table 6, if the confidence interval does not contain 0.0 , the difference is significant at the 0.05 level, and thus the difference of means of $U^{\mathrm{I}}$ is marked with an asterisk. If the confidence interval does contain 0.0 , the difference is not significant at the 0.05 level. sions:

Based on Table 6, one can make the following conclu-

(i) With respect to the proposed MSVR-PSO method, the difference in prediction performance between MSVR-PSO-W and MSVR-PSO-WO models is not significant at the 0.05 level. In addition, same conclusion can be drawn when comparing the performance between ANN-W and ANN-WO models.

(ii) The proposed MSVR-PSO method (i.e., MSVR-PSO$\mathrm{W}$ and MSVR-PSO-WO) significantly outperforms the ANN-based method, that is, ANN-W and ANNWO.

(iii) From the perspective of the modeling techniques used in this study, the four computational intelligence models (i.e., MSVR-PSO-W, MSVR-PSO-WO, ANN$\mathrm{W}$, and ANN-WO) significantly outperform the two traditional models (i.e., Holt ${ }^{\mathrm{I}}$ and VECM).

(iv) As for the comparison between two traditional models, the Holt ${ }^{\mathrm{I}}$ significantly outperforms the VECM at the 0.05 level.

\section{Conclusions}

In this study, we proposed a hybrid method, by incorporating multioutput support vector regression and particle swarm optimization (abbreviated as MSVR-PSO), for interval forecasting of the carbon futures prices. Specifically, we investigate the feasibility of forecasting the two bounds (highest and lowest prices) of carbon futures prices series simultaneously by MSVR-PSO with some potential predictors which have strong impact on carbon futures prices. The proposed MSVRPSO method and five selected competitors are developed over the period from August 12, 2010, to June 13, 2013, and their out-of-sample prediction performances are validated over the period from June 14, 2013, to November 13, 2014. According to the experimental results, two conclusions can be drawn: (1) the proposed MSVR-PSO method has the higher forecasting performance relative to five competitors, indicating that it is a promising alternative for interval forecasting of carbon futures prices; (2) introducing some potential predictors, which have strong influence on carbon futures prices, in 


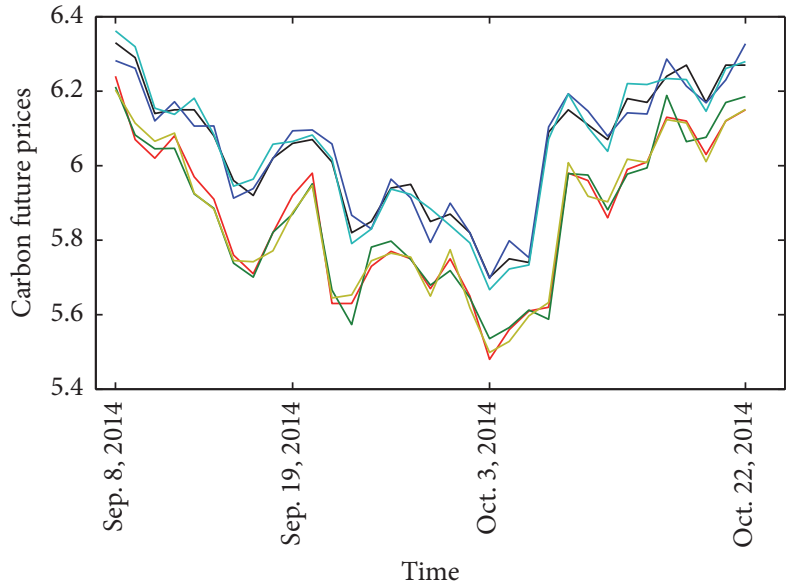

Original upper bound

- Original lower bound

_ Predicted upper bound using MSVR-PSO-WO

_ Predicted lower bound using MSVR-PSO-WO

_ Predicted upper bound using MSVR-PSO-W

_ Predicted lower bound using MSVR-PSO-W

(a)

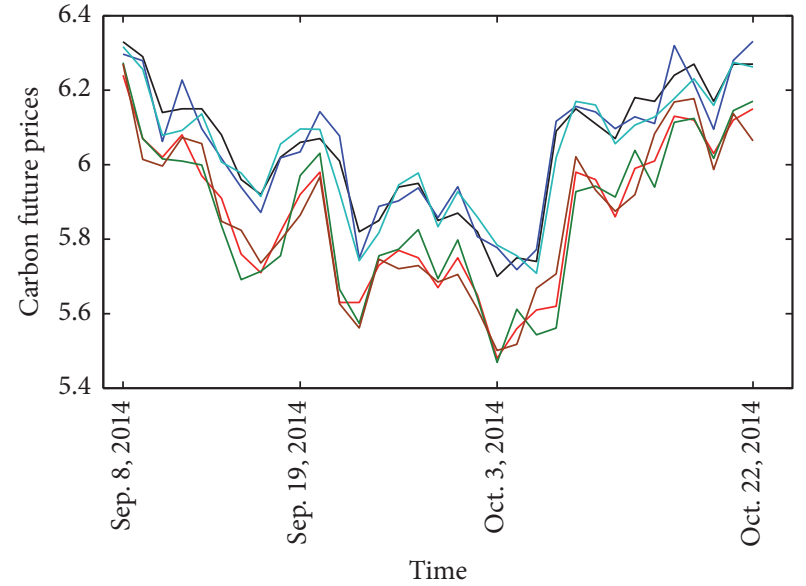

- Original upper bound

— Original lower bound

_ Predicted upper bound using ANN-W

_ Predicted lower bound using ANN-W

_ Predicted upper bound using ANN-WO

_ Predicted lower bound using ANN-WO

(b)
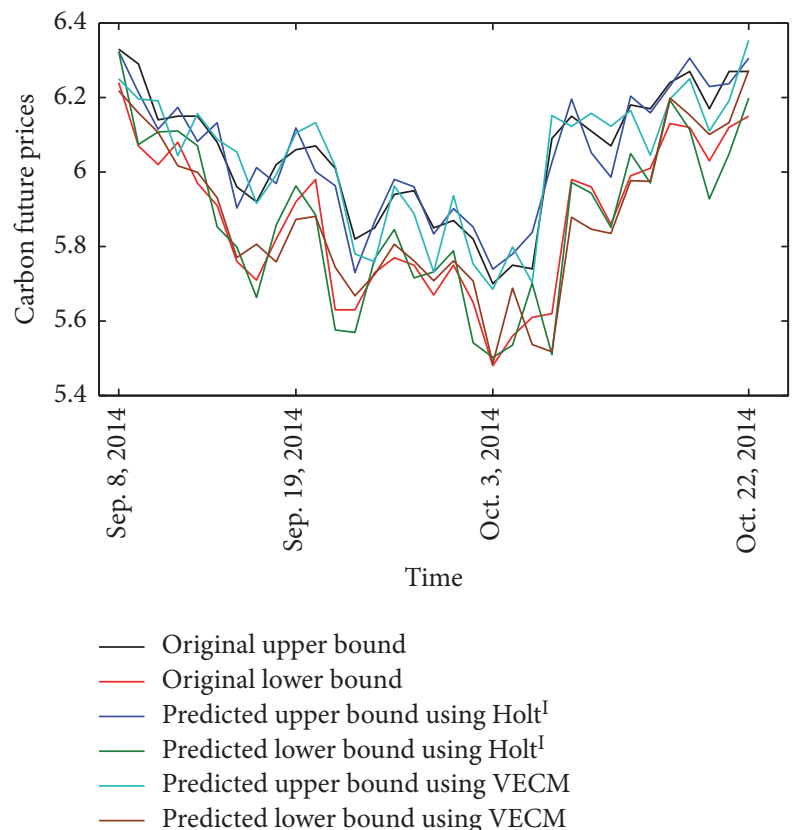

(c)

FIGURE 11: Representative examples of actual intervals versus predicted intervals using (a) MSVR-PSO-W and MSVR-PSO-WO, (b) ANN-W and ANN-WO, and (c) Holt ${ }^{\mathrm{I}}$ and VECM.

modeling process is useful for achieving better prediction performance (though lacking significance).

Our research could be extended in the following interesting directions. First, this study is restricted to one-step-ahead forecasting. Obviously, multistep-ahead forecasting could be performed to provide a more comprehensive picture. Moreover, although we examine the statistical prediction performance of all examined models, economic criteria, which are of greater value to market players in carbon futures market, should be evaluated in future research. 
TABLE 6: Multiple comparison test result.

\begin{tabular}{|c|c|c|c|c|}
\hline \multicolumn{2}{|c|}{ The two models being compared } & \multirow{2}{*}{ Lower bound of CI } & \multirow{2}{*}{ The difference of means of $U^{\mathrm{I}}$} & \multirow{2}{*}{ Upper bound of CI } \\
\hline First model & Second model & & & \\
\hline MSVR-PSO-W & MSVR-PSO-WO & -0.0314 & -0.0130 & 0.0053 \\
\hline MSVR-PSO-W & ANN-W & -0.2008 & $-0.1824^{*}$ & -0.1641 \\
\hline MSVR-PSO-W & ANN-WO & -0.2058 & $-0.1875^{*}$ & -0.1691 \\
\hline MSVR-PSO-W & Holt $^{\mathrm{I}}$ & -0.3330 & $-0.3147^{*}$ & -0.2963 \\
\hline MSVR-PSO-W & VECM & -0.3770 & $-0.3587^{*}$ & -0.3403 \\
\hline MSVR-PSO-WO & ANN-W & -0.1877 & $-0.1694^{*}$ & -0.1511 \\
\hline MSVR-PSO-WO & ANN-WO & -0.1927 & $-0.1744^{*}$ & -0.1561 \\
\hline MSVR-PSO-WO & Holt $^{\mathrm{I}}$ & -0.3199 & $-0.3016^{*}$ & -0.2833 \\
\hline MSVR-PSO-WO & VECM & -0.3639 & $-0.3456^{*}$ & -0.3273 \\
\hline ANN-W & ANN-WO & -0.0233 & -0.0050 & 0.0133 \\
\hline ANN-W & Holt $^{\mathrm{I}}$ & -0.1505 & $-0.1322^{*}$ & -0.1139 \\
\hline ANN-W & VECM & -0.1945 & $-0.1762^{*}$ & -0.1579 \\
\hline ANN-WO & Holt $^{\mathrm{I}}$ & -0.1455 & $-0.1272^{*}$ & -0.1089 \\
\hline ANN-WO & VECM & -0.1895 & $-0.1712^{*}$ & -0.1529 \\
\hline Holt $^{\mathrm{I}}$ & VECM & -0.0623 & $-0.0440^{*}$ & -0.0257 \\
\hline
\end{tabular}

Notes.* indicates that the mean difference between the two adjacent models is significant at the 0.05 level.

\section{Conflicts of Interest}

The authors declare that there are no conflicts of interest regarding the publication of this paper.

\section{Acknowledgments}

This work was supported by the Natural Science Foundation of China (Project nos. 71501079, 41501213, and 71273105), the China Postdoctoral Science Foundation (Project nos. 2015M570648 and 2015M570650), the Key Project for Studies of Philosophy and Social Sciences by Ministry of Education (15JZD014), and the Fundamental Research Funds for the Central Universities (Project nos. 2662014BQ045, 2662015PY026, and 2662017PY045).

\section{References}

[1] B. Zhu, J. Chevallier, S. Ma, and Y. Wei, "Examining the structural changes of European carbon futures price 2005-2012," Applied Economics Letters, vol. 22, no. 5, pp. 335-342, 2015.

[2] B. Zhu, D. Han, J. Chevallier, and Y.-M. Wei, "Dynamic multiscale interactions between European carbon and electricity markets during 2005-2016," Energy Policy, vol. 107, pp. 309-322, 2017.

[3] Z.-H. Feng, L.-L. Zou, and Y.-M. Wei, "Carbon price volatility: Evidence from EU ETS,” Applied Energy, vol. 88, no. 3, pp. 590598, 2011.

[4] S. J. Byun and H. Cho, "Forecasting carbon futures volatility using GARCH models with energy volatilities," Energy Economics, vol. 40, pp. 207-221, 2013.

[5] J. Chevallier, "Nonparametric modeling of carbon prices," Energy Economics, vol. 33, no. 6, pp. 1267-1282, 2011.
[6] X. Fan, S. Li, and L. Tian, "Chaotic characteristic identification for carbon price and an multi-layer perceptron network prediction model," Expert Systems with Applications, vol. 42, no. 8, pp. 3945-3952, 2015.

[7] S. K. Han, J. J. Ahn, K. J. Oh, and T. Y. Kim, "A new methodology for carbon price forecasting in EU ETS," Expert Systems, vol. 32, no. 2, pp. 228-243, 2015.

[8] G. Koop and L. Tole, "Forecasting the European carbon market," Journal of the Royal Statistical Society. Series A. Statistics in Society, vol. 176, no. 3, pp. 723-741, 2013.

[9] S. Viteva, Y. V. Veld-Merkoulova, and K. Campbell, "The forecasting accuracy of implied volatility from ECX carbon options," Energy Economics, vol. 45, pp. 475-484, 2014.

[10] B. Zhu, "A novel multiscale ensemble carbon price prediction model integrating empirical mode decomposition, genetic algorithm and artificial neural network," Energies, vol. 5, no. 2, pp. 355-370, 2012.

[11] B. Zhu and Y. Wei, "Carbon price forecasting with a novel hybrid ARIMA and least squares support vector machines methodology," Omega (United Kingdom), vol. 41, no. 3, pp. 517524, 2012.

[12] B. Zhu, D. Han, P. Wang, Z. Wu, T. Zhang, and Y.-M. Wei, "Forecasting carbon price using empirical mode decomposition and evolutionary least squares support vector regression," Applied Energy, vol. 191, pp. 521-530, 2017.

[13] K. Hong, H. Jung, and M. Park, "Predicting European carbon emission price movements," Carbon Management, vol. 8, no. 1, pp. 33-44, 2017.

[14] M. Segnon, T. Lux, and R. Gupta, "Modeling and forecasting the volatility of carbon dioxide emission allowance prices: a review and comparison of modern volatility models," Renewable and Sustainable Energy Reviews, vol. 69, pp. 692-704, 2017.

[15] G. S. Atsalakis, "Using computational intelligence to forecast carbon prices," Applied Soft Computing Journal, vol. 43, pp. 107116, 2016. 
[16] R. E. Moore, Interval Analysis, Prentice-Hall, Englewood Cliffs, NJ, USA, 1966.

[17] R. E. Moore, Methods and Applications of Interval Analysis, SIAM, Philadelphia, Pa, USA, 1979.

[18] J. Arroyo, A. Muñoz, S. Roque, C. Maté, and A. Sarabia, "Exponential smoothing methods for interval time series," in Proceedings of the 1st European Symposium on Time Series Prediction, pp. 231-240, 2007.

[19] L. Billard and E. Diday, "Regression analysis for interval-valued data," in Proceedings of the Conference of the International Federation of Classification Societies, pp. 369-374, SpringerVerlag, Berlin, Germany, 2000.

[20] J. Arroyo, R. Espínola, and C. Maté, "Different Approaches to Forecast Interval Time Series: A Comparison in Finance," Computational Economics, vol. 37, no. 2, pp. 169-191, 2011.

[21] C. Hu and L. T. He, "An application of interval methods to stock market forecasting," Reliable Computing. An International Journal Devoted to Reliable Mathematical Computations Based on Finite Representations and Guaranteed Accuracy, vol. 13, no. 5, pp. 423-434, 2007.

[22] T. Xiong, Y. Bao, and Z. Hu, "Multiple-output support vector regression with a firefly algorithm for interval-valued stock price index forecasting," Knowledge-Based Systems, vol. 55, pp. 87-100, 2014.

[23] A. Han, Y. Hong, K. K. Lai, and S. Wang, "Interval time series analysis with an application to the sterling-dollar exchange rate," Journal of Systems Science \& Complexity, vol. 21, no. 4, pp. 558573,2008

[24] W. Yang, A. Han, K. Cai, and S. Wang, "ACIX model with interval dummy variables and its application in forecasting interval-valued crude oil prices," in Proceedings of the 12th Annual International Conference on Computational Science, ICCS 2012, pp. 1273-1282, June 2012.

[25] T. Xiong, Y. Bao, and Z. Hu, "Interval forecasting of electricity demand: a novel bivariate EMD-based support vector regression modeling framework," International Journal of Electrical Power and Energy Systems, vol. 63, pp. 353-362, 2014.

[26] C. García-Ascanio and C. Maté, "Electric power demand forecasting using interval time series: A comparison between VAR and iMLP," Energy Policy, vol. 38, no. 2, pp. 715-725, 2010.

[27] A. M. S. Roque, C. Maté, J. Arroyo, and Á. Sarabia, "IMLP: Applying multi-layer perceptrons to interval-valued data," Neural Processing Letters, vol. 25, no. 2, pp. 157-169, 2007.

[28] S. B. Taieb, G. Bontempi, A. Sorjamaa, and A. Lendasse, "Long-term prediction of time series by combining direct and MIMO strategies," in Proceedings of the 2009 International Joint Conference on Neural Networks, IJCNN 2009, pp. 3054-3061, June 2009.

[29] J. Kennedy and R. Eberhart, "Particle swarm optimization," in Proceedings of the IEEE International Conference on Neural Networks, vol. 4, pp. 1942-1948, Perth, Australia, December 1995.

[30] A. L. S. Maia and F. D. A. T. de Carvalho, "Holt's exponential smoothing and neural network models for forecasting intervalvalued time series," International Journal of Forecasting, vol. 27, no. 3, pp. 740-759, 2011.

[31] Y.-L. Cheung, Y.-W. Cheung, and A. T. Wan, "A high-low model of daily stock price ranges," Journal of Forecasting, vol. 28, no. 2, pp. 103-119, 2009.

[32] F. Pérezcruz, G. Campsvalls, E. Soriaolivas, J. J. Pérezruixo, A. R. Figueirasvidal, and A. Artésrodríguez, "Multi-dimensional function approximation and regression estimation," in Proceedings of the International Conference on Artificial Neural Networks, pp. 757-762, 2002.

[33] M. Sanchez-Fernandez, M. de-Prado-Cumplido, J. ArenasGarcia, and F. Perez-Cruz, "SVM multiregression for nonlinear channel estimation in multiple-input multiple-output systems," IEEE Transactions on Signal Processing, vol. 52, no. 8, pp. 22982307, 2004.

[34] D. Tuia, J. Verrelst, L. Alonso, F. Perez-Cruz, and G. CampsValls, "Multioutput support vector regression for remote sensing biophysical parameter estimation," IEEE Geoscience and Remote Sensing Letters, vol. 8, no. 4, pp. 804-808, 2011.

[35] T. Xiong, C. Li, Y. Bao, Z. Hu, and L. Zhang, "A combination method for interval forecasting of agricultural commodity futures prices," Knowledge-Based Systems, vol. 77, pp. 92-102, 2015.

[36] B. Scholkopf and A. J. Smola, Learning with Kernels: Support Vector Machines, Regularization, Optimization, and Beyond, MIT Press, 2001.

[37] E. Alberola, J. Chevallier, and B. Chèze, "Price drivers and structural breaks in European carbon prices 2005-2007," Energy Policy, vol. 36, no. 2, pp. 787-797, 2008.

[38] M. M. Bataller, J. Chevallier, M. Hervé-Mignucci, and E. Alberola, "The EUA-sCER spread: compliance strategies and arbitrage in the European carbon market," SSRN Electronic Journal, vol. 39, pp. 1056-1069, 2010.

[39] B. Hintermann, "Allowance price drivers in the first phase of the EU ETS," Journal of Environmental Economics and Management, vol. 59, no. 1, pp. 43-56, 2010.

[40] F. Ramsay and D. Schaefer, The Statistical Sleuth, Duxbury, Boston, Mass, USA, 1996.

[41] Y. Bao, T. Xiong, and Z. Hu, "PSO-mismo modeling strategy for multistep-ahead time series prediction," IEEE Transactions on Cybernetics, vol. 44, no. 5, pp. 655-668, 2014.

[42] G. Rubio, H. Pomares, I. Rojas, and L. J. Herrera, "A heuristic method for parameter selection in LS-SVM: application to time series prediction," International Journal of Forecasting, vol. 27, no. 3, pp. 725-739, 2011.

[43] Y. Lu, N. Zeng, X. Liu, and S. Yi, "A new hybrid algorithm for bankruptcy prediction using switching particle swarm optimization and support vector machines," Discrete Dynamics in Nature and Society, vol. 2015, Article ID 294930, 7 pages, 2015.

[44] Z. Yang, C. Liu, X. Wang, and W. Qian, "An improved multiobjective PSO for the scheduling problem of panel block construction," Discrete Dynamics in Nature and Society, vol. 2016, Article ID 5413520, 2016. 


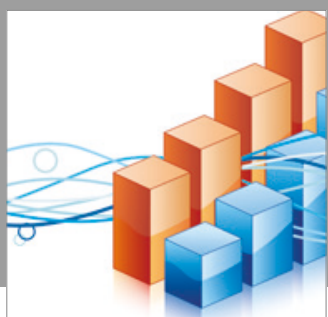

Advances in

Operations Research

vatersals

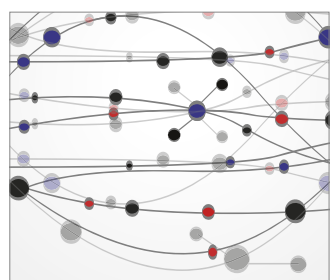

\section{The Scientific} World Journal
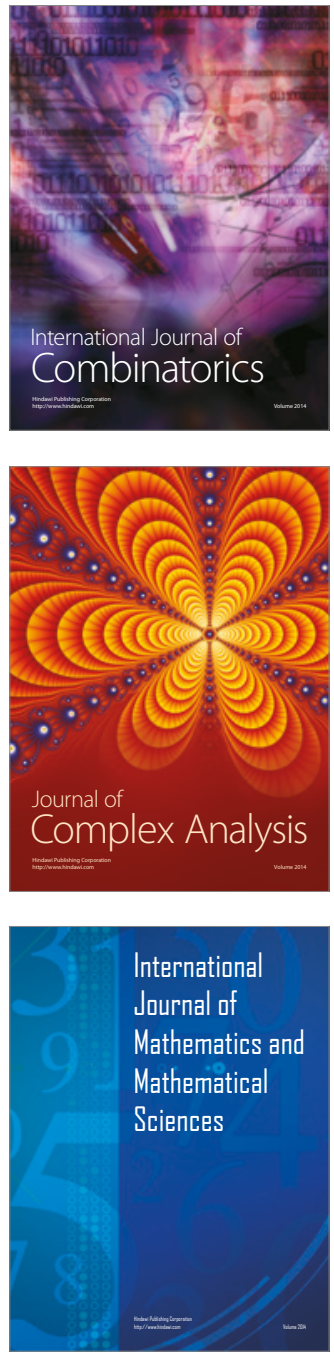
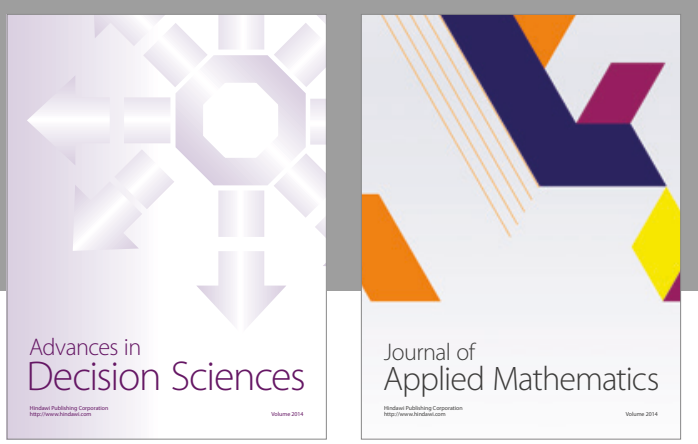

Algebra

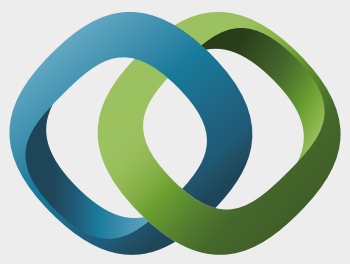

\section{Hindawi}

Submit your manuscripts at

https://www.hindawi.com
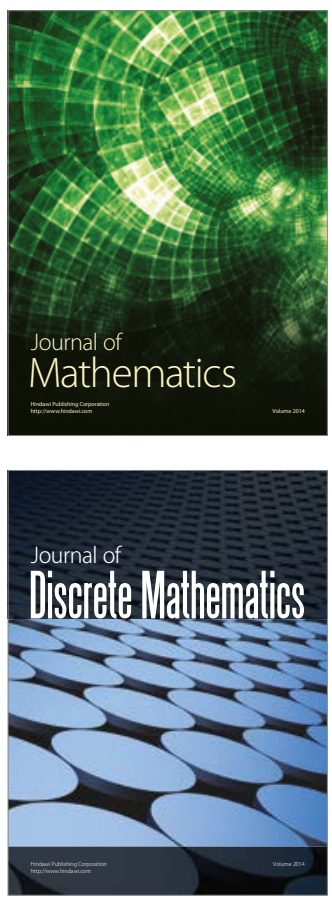

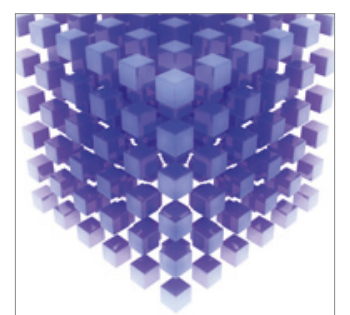

Mathematical Problems in Engineering
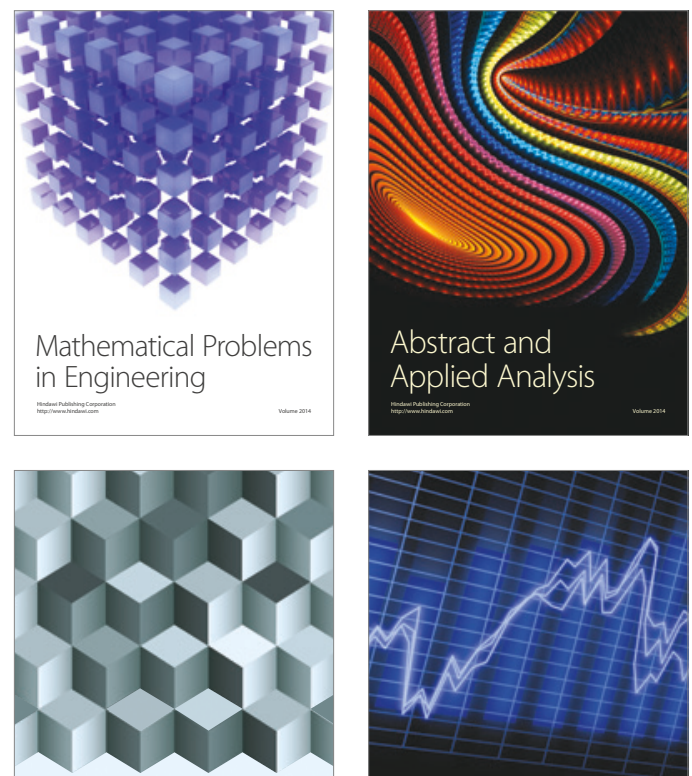

Journal of

Function Spaces

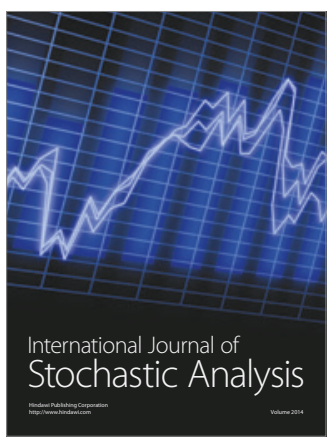

Probability and Statistics
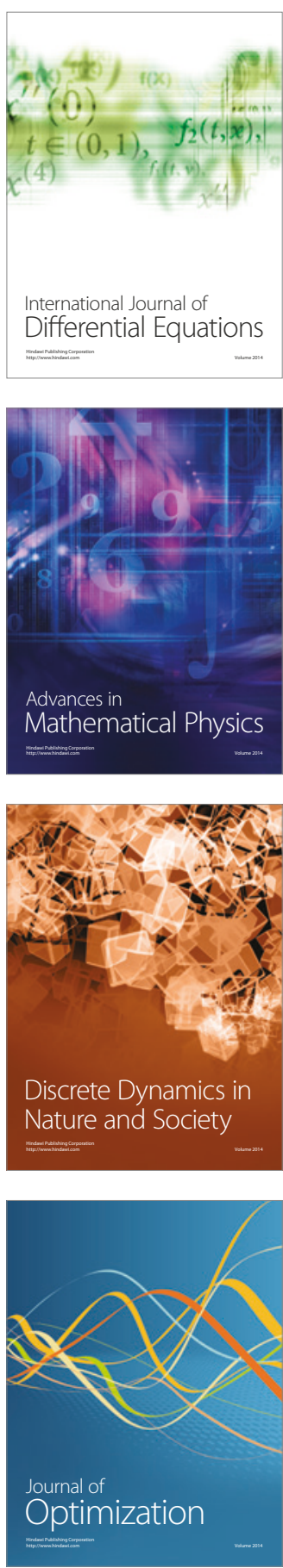